\title{
Non-Contrast-Enhanced Silent Scan MR Angiography of Intracranial Anterior Circulation Aneurysms Treated with a Low-Profile Visualized Intraluminal Support Device
}

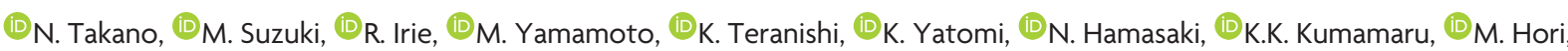
(D) H. Oishi, and DS. Aoki

\begin{abstract}
BACKGROUND AND PURPOSE: The Low-Profile Visualized Intraluminal Support Device comprises a small-cell nitinol structure and a single-wire braided stent that provides greater metal coverage than previously reported intracranial stents, as well as assumed strong susceptibility artifacts. This study aimed to assess the benefits of non-contrast-enhanced MRA by using a Silent Scan (Silent MRA) for intracranial anterior circulation aneurysms treated with Low-Profile Visualized Intraluminal Support Device stents.
\end{abstract}

MATERIALS AND METHODS: Thirty-one aneurysms treated with Low-Profile Visualized Intraluminal Support Device stents were assessed by using Silent MRA, 3D TOF-MRA, and x-ray DSA. The quality of MRA visualization of the reconstructed artery was graded on a 4-point scale from 1 (not visible) to 4 (excellent). Aneurysm occlusion status was evaluated by using a 2-grade scale (total occlusion/remnant [neck or aneurysm]). Weighted $\kappa$ statistics were used to evaluate interobserver and intermodality agreement.

RESULTS: The mean scores \pm SDs for Silent MRA and 3D TOF-MRA were $3.16 \pm 0.79$ and $1.48 \pm 0.67(P<.05)$, respectively, with substantial interobserver agreement $(\kappa=0.66)$. The aneurysm occlusion rates of the 2-grade scale (total occlusion/remnant [neck or aneurysm]) were $69 \% / 31 \%$ for DSA, $65 \% / 35 \%$ for Silent MRA, and $92 \% / 8 \%$ for 3D TOF-MRA, respectively. The intermodality agreements were 0.88 and 0.30 for DSA/Silent MRA and DSA/3D TOF-MRA, respectively.

CONCLUSIONS: Silent MRA seems to be useful for visualizing intracranial anterior circulation aneurysms treated with Low-Profile Visualized Intraluminal Support Device stents.

ABBREVIATIONS: $\quad$ CE = contrast-enhanced; LVIS Jr. = Low-Profile Visualized Intraluminal Support Device; UTE = ultrashort TE

$\mathbf{E}^{\mathrm{n}}$ ndovascular treatment involving coil embolization is commonly used to manage both ruptured and unruptured intracranial aneurysms. ${ }^{1,2}$ In recent years, intracranial stents, including the Neuroform ${ }^{3,4}$ (Stryker, Kalamazoo, Michigan) and Enterprise stents $^{5,6}$ (Codman \& Shurtleff, Raynham, Massachusetts), have been implemented for the stent-assisted coil embolization of wide-neck

Received December 15, 2016; accepted after revision March 14, 2017.

From the Department of Radiology (N.T., M.S., K.K.K., M.H., S.A.), Graduate School of Medicine, Juntendo University, Tokyo, Japan; Department of Radiology (N.T., M.S., R.I., N.H., K.K.K., M.H., S.A.), Juntendo University Hospital, Tokyo, Japan; and Departments of Neurosurgery (M.Y., K.T., K.Y., H.O.) and Neuroendovascular Therapy (H.O.), Juntendo University School of Medicine, Tokyo, Japan.

This work was funded by the Impulsing Paradigm Change through disruptive Technologies Program of the Council for Science, Technology, and Innovation (Cabinet Office, Government of Japan). This work was also supported by the Japan Society for the Promotion of Science Grants-in-Aid for Scientific Research Grant No. JP16H06280 and Grant-in-Aid for Scientific Research on Innovative Areas, resource and technical support platforms for promoting research "Advanced Bioimaging Support."

Please address correspondence to Nao Takano, RT, MMSc, Department of Radiology, Graduate School of Medicine, Juntendo University, 2-1-1 Hongo, Bunkyo-ku,

Tokyo 113-8421, Japan; e-mail: ntakano@juntendo.ac.jp

- Indicates open access to non-subscribers at www.ajnr.org

http://dx.doi.org/10.3174/ajnr.A5223 aneurysms. Both types of stents comprise nitinol and are self-expandable. The Neuroform stent features an open-cell design with stainless steel and platinum markers, ${ }^{7,8}$ whereas the Enterprise stent is a closed-cell design with tantalum markers. ${ }^{9,10}$

This study implemented the Low-Profile Visualized Intraluminal Support Device ${ }^{1-14}$ (LVIS Jr. stent; MicroVention, Tustin, California), a self-expandable nitinol single-wire braided stent comprising $1.5-\mathrm{mm}$ cells. The LVIS Jr. stent contains 3 radiopaque tantalum markers on the proximal and distal tines and 3 tantalum helical strands in the stent body ${ }^{13,14}$; thus, it provides higher metal coverage $(12 \%-21 \%)^{11-13}$ relative to previously reported stents. Accordingly, stent-related magnetic susceptibility during MR imaging is a topic of research interest.

Despite reports of the use of 3D TOF-MRA after stent-assisted coil embolization, ${ }^{9}$ the radiofrequency shielding and susceptibility artifacts make it difficult to visualize flow in an intracranial stent with this technique. Because the LVIS Jr. stent is composed of nitinol and features a braided component and higher metal coverage relative to earlier stents, this device may not facilitate depiction of an intracranial stent with 3D TOF-MRA for radiofrequency shielding and susceptibility artifacts. 


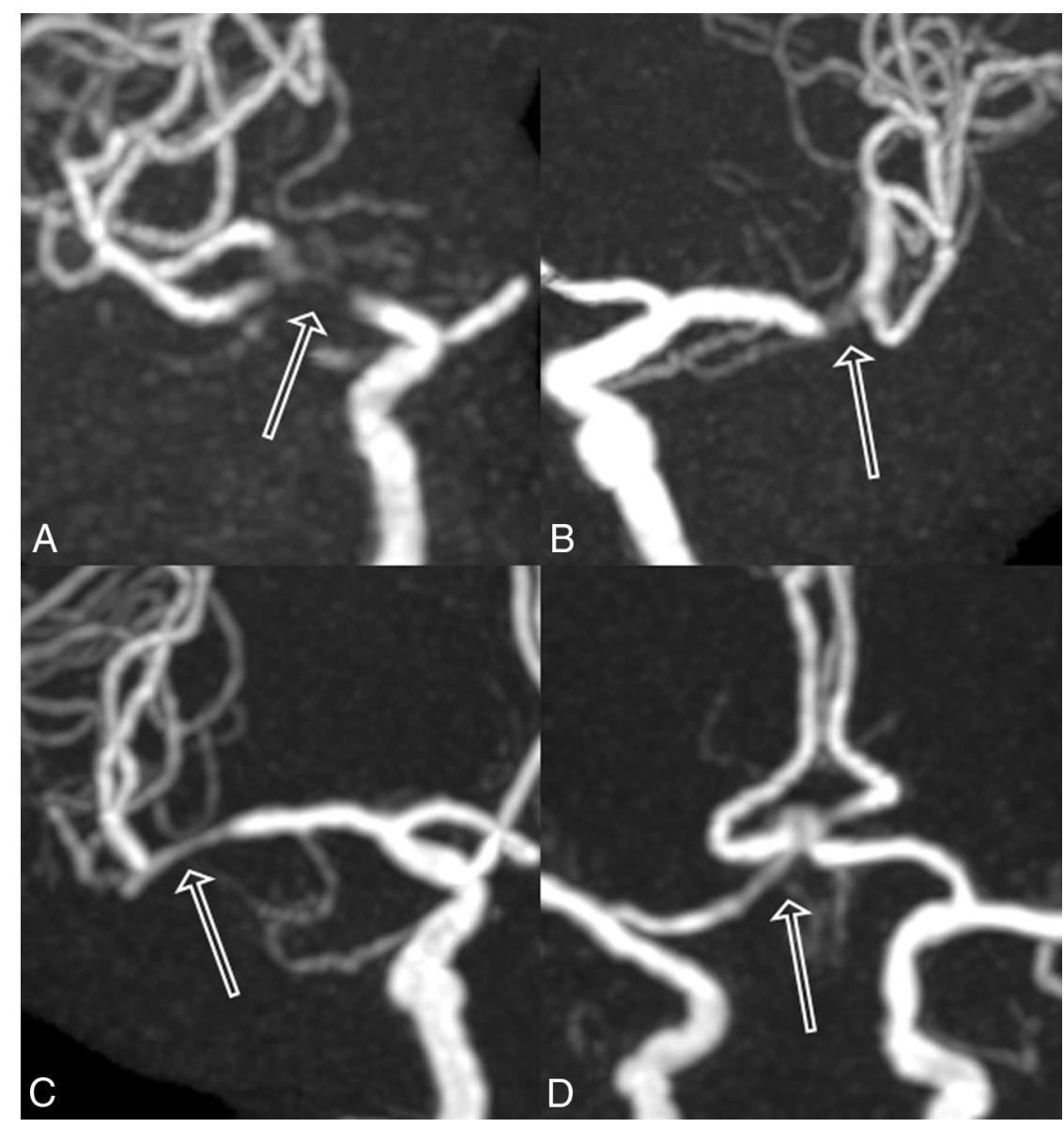

FIG 1. Examples of MRA scoring. The MRA scores by 2 observers were classified on a 4-point scale as follows: 1, (A); 2, (B); 3, (C); 4, (D). Outlined arrows are the stented segment.

used in 31 aneurysms). We deployed the following stent sizes (diameter $\times$ length): $2.5 \times 13 \mathrm{~mm}(n=21) ; 2.5 \times 17 \mathrm{~mm}(n=$ $14) ; 2.5 \times 23 \mathrm{~mm}(n=3)$; and $3.5 \times 18$ $\mathrm{mm}, 3.5 \times 23 \mathrm{~mm}$, and $3.5 \times 28 \mathrm{~mm}(n=$ 1 each). The distribution of aneurysm locations was as follows: anterior communicating artery $(n=15)$, internal carotid artery $(n=3)$, and middle cerebral artery $(n=13)$.

For all patients, Silent MRA and 3D TOF-MRA were performed in a single scanning session. The average interval between aneurysm treatment and both MRA evaluations was 5.6 days (range, 1-83 days; median, 1 day). The average interval between the most recent DSA evaluation and both MRA evaluations was 1.6 days (range, 1-9 days; median, 1 day).

\section{Silent MRA}

Silent MRA was performed with a Silent Scan. However, the details of the Silent Scan algorithm were undisclosed. Silent Scan is a combination of a 3D acquisition and reconstruction technology. This technique requires highly stable system power electronics (gradients and radiofrequency) and fast radiofrequency switching capability within the radiofrequency coil structure.

This process slowly steps the gradi-

Silent Scan (GE Healthcare, Milwaukee, Wisconsin) MRA (Silent MRA) is a non-contrast-enhanced MRA technique that uses an ultrashort TE (UTE) combined with arterial spin-labeling, ${ }^{15}$ which is used as a preparation pulse to visualize blood flow. Subsequent data acquisition is based on 3D radial sampling. ${ }^{15,16} \mathrm{Be}-$ cause UTE decreases the susceptibility artifacts associated with metallic devices, Silent MRA could potentially reduce the artifacts associated with braided, high-metal-coverage stents. Accordingly, we evaluated the efficacy of Silent MRA versus 3D TOF-MRA for intracranial anterior circulation aneurysms treated with LVIS Jr. stent-assisted coil embolization.

\section{MATERIALS AND METHODS \\ Patient and Aneurysm Characteristics}

Between July 2015 and September 2016, 32 aneurysms in 29 patients treated with LVIS Jr. stent-assisted coil embolization for anterior circulation aneurysms were retrospectively examined. We obtained approval for this study from the ethics review board. Written informed consent was not required because of the retrospective nature of this study. One patient was excluded from the study due to postoperative deep brain stimulation with an MR imagingunsafe device. The remaining 28 patients with 31 aneurysms comprised 13 men and 15 women with a mean age of $60.8 \pm 11.3$ years (range, 41-77 years). Twenty-one aneurysms were treated with a single stent, and 10 were treated with 2 stents (ie, 41 LVIS Jr. stents were ents while collecting data during a 3D acquisition. The data acquisition is based on a $3 \mathrm{D}$ radial sampling, and an arterial spinlabeling technique is used as a preparation pulse to visualize blood flow. In Silent MRA, control images (nonlabelled images) are scanned before the labeling pulse. Labeled images are scanned after the labeling pulse, and the labeled blood exhibits low signal intensity. The angiographic images of blood flow are the result of subtraction of labeled images from control images.

\section{Intra-Arterial DSA Technique}

$\mathrm{X}$-ray catheter-based intra-arterial cerebral DSA was performed with the following biplane angiographic system: Artis Q BA Twin (Siemens, Erlangen, Germany). The image matrix and FOV were $1024 \times 1024$ and $170 \times 170 \mathrm{~mm}$, respectively. The temporal resolution was 3 frames per second. Selective manual internal carotid artery injections were administered according to the aneurysm location. Rotational 3D angiography was performed for additional confirmation of the findings. Additional selected working angles were obtained to clarify the aneurysmal anatomy at the discretion of the neurosurgical interventionist.

\section{MRA Scan Parameters}

Silent MRA and 3D TOF-MRA were performed on a 3T MR imaging unit (Discovery MR750w; GE Healthcare) with a 12-channel 


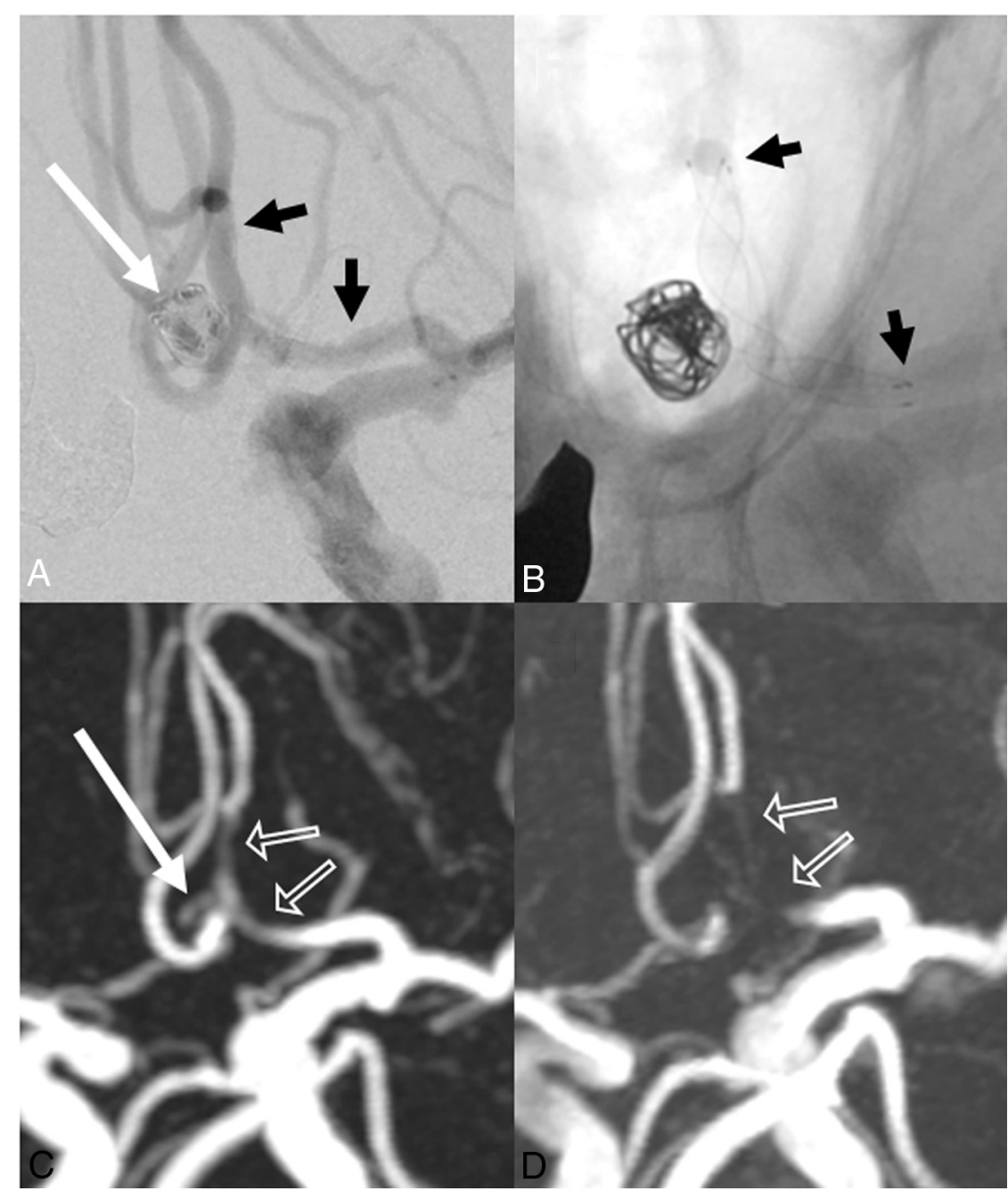

FIG 2. A 73-year-old woman. A, Stent-assisted coil embolization of the anterior communicating artery was performed with a LVIS Jr. stent $(2.5 \times 17 \mathrm{~mm})$. The aneurysm remnant is indicated by the long white arrow. Short black arrows indicate stent edges. B, X-ray digital angiography reveals stent edges (short black arrows). C, Silent MRA shows minimal signal loss at the stented segment (outlined arrows). The aneurysm remnant is visible (long white arrow). D, 3D time-of-flight MRA shows complete signal losses at the stented segments (outlined arrows). The aneurysm remnant is not depicted. The scores of Silent MRA by 2 observers are 4 and 4 . The scores of 3D TOF-MRA are 1 and 1.

head-neck coil system. The following scan parameters were used for Silent MRA: TR/TE, 642.8/0.016 ms; flip angle, $5^{\circ}$; FOV, $180 \times$ $180 \mathrm{~mm}$; matrix, $180 \times 180$; section thickness, $1.0 \mathrm{~mm}$ (with section interpolation); NEX, 1.4; bandwidth, $\pm 31.2 \mathrm{kHz}$; acquisition time, 12 minutes 13 seconds; and reconstructed voxel size, $1.0 \times 1.0 \times 0.5 \mathrm{~mm}$.

The 3D TOF-MRA scan parameters were as follows: TR/TE, 20/2.9 ms; flip angle, $18^{\circ}$; FOV, $200 \times 200 \mathrm{~mm}$; acquisition matrix, $416 \times 224$; section thickness, $1.0 \mathrm{~mm}$ (with section interpolation); NEX, 1; bandwidth, $\pm 41.7 \mathrm{kHz}$; acquisition time, $4 \mathrm{~min}$ utes 45 seconds ( 3 slabs, overlap between a slab: 14 sections, 1 slab: 38 sections); reconstruction matrix, 512; and reconstructed voxel size, $0.39 \times 0.39 \times 0.5 \mathrm{~mm}$.

\section{Image Analysis}

Each DSA, Silent MRA, and 3D TOF-MRA series was anonymized by random number assignment. We evaluated DSA images and the 2 types of MRA images. The 2 types of MRA images were assessed separately to minimize bias due to knowledge of the results of the other MRA image. We used MIP and source images. All images were independently evaluated in random order by 2 neuroradiologists (M.S. and R.I., who have 15 and 6 years of experience in neuroradiology, respectively) on the PACS. In all images, the window-width and windowlevel could be modified for evaluation. The locations of the treated aneurysms to be evaluated were provided to the 2 observers because the pretreatment $\mathrm{x}$-ray DSA images were unavailable.

The quality of Silent MRA- and 3D TOF-MRA-based visualization of the reconstructed arteries was evaluated. Two neuroradiologists independently reviewed both types of MRAs and rated visualization of the flow in each stent subjectively on a 4-point scale as follows: 1 , not visible (near-complete signal loss or no signal); 2, poor (structures are slightly visible but with substantial artifacts or blurring); 3, acceptable (acceptable diagnostic quality with medium artifacts or blurring); 4, excellent (good diagnostic quality with minimal artifacts or blurring). Figure 1 shows a sample of the 4-point scale. Aneurysm occlusion was evaluated by using a 2-grade scale (total occlusion/remnant [neck or aneurysm]). The most recent DSA images were used as a reference standard.

\section{Statistical Analysis}

The scores of MIP images from the 2 observers were averaged, and the scores were compared between techniques by using the Wilcoxon signed rank test. A $P$ value $<.05$ was statistically significant. Weighted $\kappa$ statistics were used to evaluate interobserver and intermodality agreement. According to Landis and Koch, ${ }^{17} \kappa$ was interpreted as follows: $<0$, no agreement; $0-0.19$, poor agreement; $0.20-0.39$, fair agreement; $0.40-$ 0.59 , moderate agreement; $0.60-0.79$, substantial agreement; and $0.80-1.00$, almost perfect agreement. All statistical analyses were performed by using SPSS software, Version 22 (IBM, Armonk, New York).

\section{RESULTS}

The mean scores ( \pm SDs) for Silent MRA and 3D TOF-MRA differed significantly, with respective values of $3.16 \pm 0.79$ and $1.48 \pm 0.67$ $(P<.05)$, and substantial interobserver agreement was observed $(\kappa=0.66)$. The aneurysm occlusion rates of the 2-grade scale (total occlusion/remnant [neck or aneurysm]) were $69 \% / 31 \%$ for DSA, $65 \% / 35 \%$ for Silent MRA, and $92 \% / 8 \%$ for 3D TOF-MRA. Intermodality agreement values of 0.88 and 0.30 were obtained for DSA/ Silent MRA and DSA/3D TOF-MRA, respectively. Figures 2-4 are representative images. 


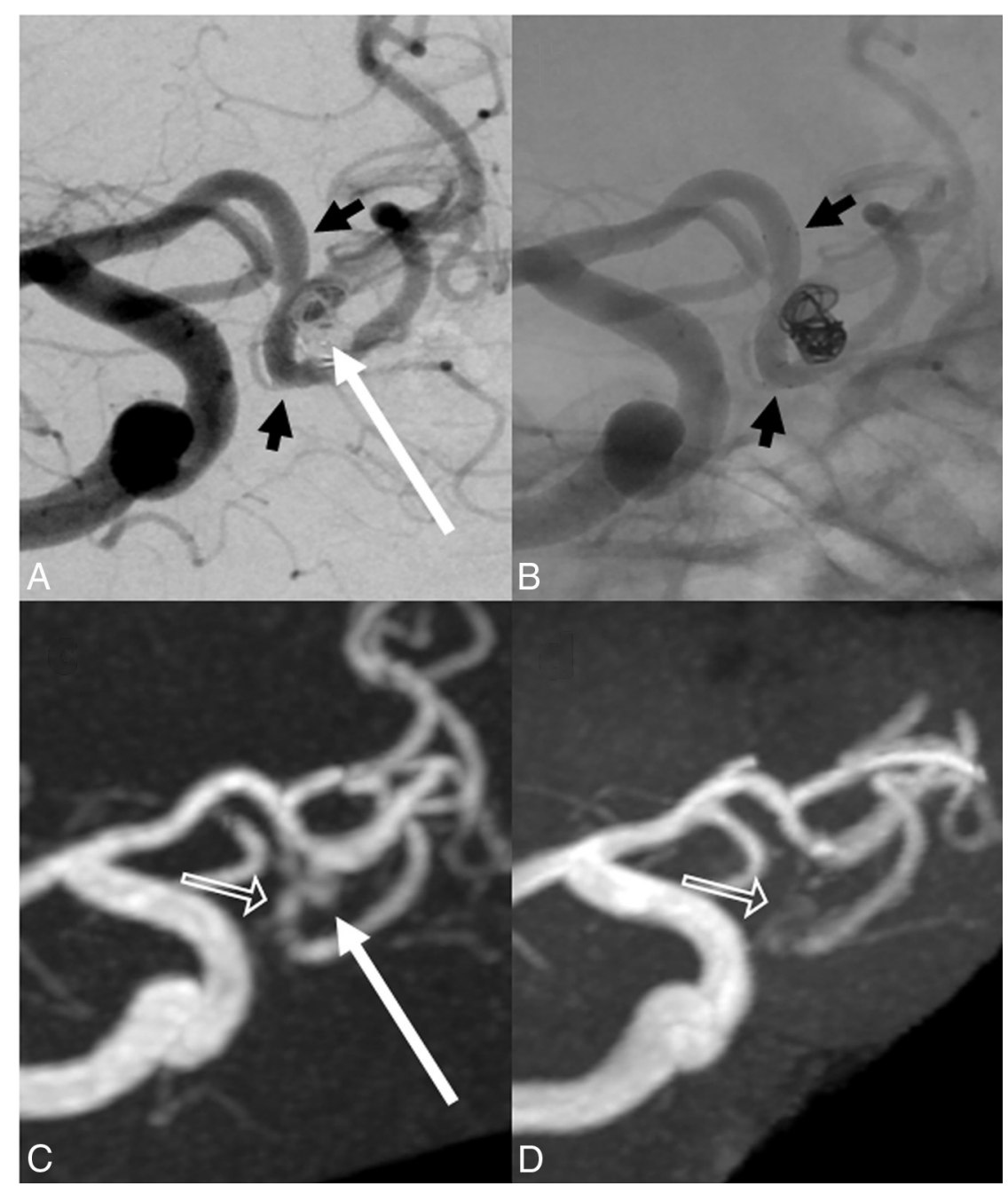

FIG 3. A 71-year-old man. A, Stent-assisted coil embolization of the anterior communicating artery is performed with a LVIS Jr. stent $(2.5 \times 17 \mathrm{~mm})$. The neck remnant is indicated by the long white arrow. Short black arrows indicate stent edges. B, X-ray digital angiography shows the stent edges (short black arrows). C, Silent MRA shows minimal signal loss at the stented segment (outlined arrow). The neck remnant is depicted almost equally well as with digital subtraction angiography (long white arrow). D, 3D time-of-flight MRA shows almost complete signal loss in the stented segment (outlined arrow). The neck remnant is not depicted. The scores of Silent MRA by 2 observers are 4 and 4 . The scores of 3D TOF-MRA are 1 and 1.

\section{DISCUSSION}

This study demonstrated superior visualization of the reconstructed artery with Silent MRA compared with 3D TOF-MRA in patients treated with LVIS Jr. stents. With consensual x-ray DSA as a reference standard, the intermodality agreement was better with Silent MRA than with 3D TOF-MRA. Moreover, Silent MRA showed strong concordance with DSA for the detection of occluded aneurysms, despite the small diameter of the LVIS Jr. stent.

Several studies have evaluated LVIS Jr. stent-assisted coil embolization $^{11-14}$; however, none have used MRA for follow-up evaluations after treatment with LVIS Jr. stents, though 3D TOF-MRA, Silent MRA, and contrast-enhanced (CE)-MRA have been used to follow up patients who underwent other stent-assisted coil embolizations. ${ }^{7,9,10,15,18} \mathrm{Cho}$ et $\mathrm{al}^{9}$ reported a good correlation between 3D rotational angiography and 3D TOF-MRA, which was attributed to the very small voxel size, whereas Irie et $\mathrm{al}^{15}$ reported the efficacy of Silent MRA in stent-assisted coil embolization. Although the authors reported superior visualization of neck remnants with Silent MRA versus 3D TOF-MRA, they assessed only closed-cell Enterprise stents. Takano et $\mathrm{al}^{19}$ reported on a Silent MRA study in Yconfiguration stent-assisted coil embolization. The visualization ability of Silent MRA was superior to that of 3D TOFMRA in a study that included Neuroform, Enterprise, and LVIS Jr. stents. In this study, Silent MRA showed neck remnants more precisely than did 3D TOFMRA. In contrast, Agid et $\mathrm{al}^{7}$ reported that CE-MRA better depicted flow in a stent, compared with 3D TOF-MRA, in a study that included Neuroform, Enterprise, and LEO (Balt Extrusion, Montmorency, France) stents. CE-MRA and DSA had similar visualization abilities; however, CE-MRA was associated with several complications such as nephrogenic systemic fibrosis, ${ }^{20}$ gadolinium accumulation, ${ }^{21}$ and anaphylactic shock.

In this study, although Silent MRA had a larger matrix size than 3D TOFMRA, it yielded superior visualization of the reconstructed artery. This outcome was attributed to the use of a UTE, which minimizes phase dispersion of the labeled blood flow signal and reduces magnetic susceptibility artifacts. Specifically, the TE was $2.9 \mathrm{~ms}$ during 3D TOF-MRA, but only $0.016 \mathrm{~ms}$ during Silent MRA. Choi et $\mathrm{al}^{8}$ reported that a short TE could increase signal intensity while optimizing the stent-visualization parameter. Similarly, Yamada et $\mathrm{al}^{22}$ reported that a TE of $1.54-1.60 \mathrm{~ms}$ was used in their 3D TOF-MRA study, and Ikushima et $\mathrm{al}^{23}$ reported that a wide bandwidth (short TE) could increase the in-stent signal intensity and in-coil signal intensity during a CE-MRA phantom study. Furthermore, Irie et $\mathrm{al}^{15}$ reported a TE of $0.016 \mathrm{~ms}$ during Silent MRA. In our study, Silent MRA with a UTE allowed the precise visualization of instent flow.

A LVIS Jr. stent diameter of $2.5 \mathrm{~mm}$ was used for 38 stent placements in 28 patients (93\%). During 3D TOF-MRA, the instent signal in this type of stent is affected by susceptibility artifacts caused by stents and coils. Therefore, the use of a smalldiameter stent is more likely to reduce the in-stent signal. In contrast, Silent MRA allows precise visualization of the in-stent signal. The UTE used with this technique can minimize phase dispersion of the labeled blood flow signal in the voxel and reduce the magnetic susceptibility artifacts. Accordingly, artifacts caused by stents or coils are reduced.

Notably, Silent MRA yields better detection of aneurysm occlusions compared with 3D TOF-MRA. Because 3D TOF-MRA uses the inflow effect to visualize blood flow signal, a higher flip 


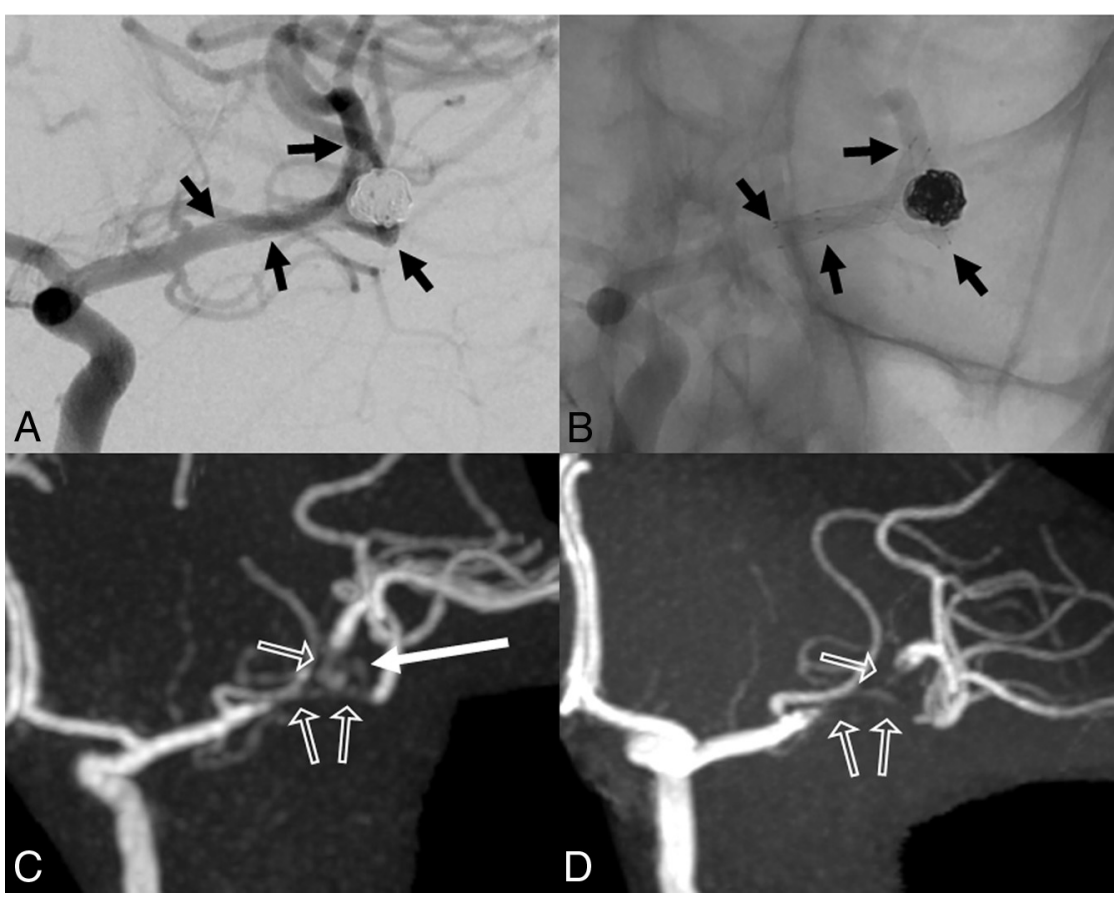

FIG 4. A 45-year-old woman. $A$, Stent-assisted coil embolization is performed with LVIS Jr. stents $(2.5 \times 13 \mathrm{~mm}$ and $2.5 \times 17 \mathrm{~mm})$ for a left middle cerebral artery bifurcation. The neck or aneurysm remnant is not depicted. Short black arrows indicate stent edges. B, X-ray digital angiography indicates stent edges (short black arrows). C, Silent MRA depicts the aneurysm remnant (long white arrow). Stented segments are slightly visible, and moderate signal loss is apparent on Silent MRA (outlined arrows). D, 3D time-of-flight MRA does not depict the neck or aneurysm remnant and yields a complete signal loss at the stented segment (outlined arrows). The scores of Silent MRA by 2 observers are 3 and 2. The scores of 3D TOF-MRA are 1 and 1 .

angle is needed to increase the signal intensity ${ }^{8,24,25}$; however, in areas of slow or turbulent flow, the saturation effect caused by a high flip angle decreases the signal intensity. Therefore, visualization of the neck remnant is difficult, even if the higher flip angle increases the signal intensity in the stent. In contrast, Silent MRA yields good visualization of the neck remnant because it uses arterial spin-labeling and UTE for blood flow depiction and phase dispersion, respectively.

Most interesting, in our series, a helmet-type remnant ${ }^{26}$ was detected in 3 patients by Silent MRA. This remnant type is caused by a lack of $\mathrm{x}$-ray penetration of the coil mass itself. According to Agid et al, ${ }^{26}$ CE-MRA is superior to $\mathrm{x}$-ray DSA for the detection of helmet-type remnants. Notably, Silent MRA may similarly detect helmet-type remnants and has the advantage of being a non-contrast-enhanced technique. Figure 4 demonstrates a helmet-type remnant.

The self-expandable, single-wire braided nature of the LVIS Jr. stent allows greater metal coverage of the blood vessel, compared with conventional open-cell and closed-cell stents. The open-cell Neuroform stent and closed-cell Enterprise stent yield $11 \%$ and $10 \%$ coverage, respectively, ${ }^{14}$ and flow-diverter stents yield approximately $30 \%-35 \%{ }^{27}$ coverage. In contrast, the LVIS Jr. stent yields approximately $12 \%-21 \%$ coverage. ${ }^{1-13}$ Furthermore, the adaptation blood vessel diameter is smaller with the LVIS Jr. stent compared with the Neuroform and Enterprise stents. Because the metal quantity per unit blood vessel area increases with the LVIS Jr. stent, 3D TOF-
MRA depictions of the neck remnants of aneurysms are thought to be more difficult following LVIS Jr. stent placement, compared with Neuroform and Enterprise stent placement.

Nevertheless, our study observed a good intermodality agreement between DSA and Silent MRA during an evaluation of aneurysm occlusion. In particular, Silent MRA and x-ray DSA yielded results of similar quality, a finding that was attributed to the reduction in susceptibility artifacts as a result of using a UTE during Silent MRA. In addition, the blood flow saturation effect was reduced when using arterial spin-labeling for blood flow depiction. Thus, Silent MRA might be useful for follow-ups of patients treated with LVIS Jr. stents.

Silent MRA can be used to visualize the in-stent flow. Moreover, it can depict a neck remnant also shown with $\mathrm{x}$ ray DSA. Therefore, Silent MRA can be used as a substitute for the X-ray DSA.

One of the disadvantages of Silent MRA is motion artifacts due to the long scanning duration. However, only a few cases showed severe motion artifacts. In many cases, the artifacts were caused by an oral motion, so there was not much influence on the depiction of intracranial blood vessels. The acquisition time required for Silent MRA is very long for clinical use, but it offers advantages in the visualization of in-stent flow.

Our study had some limitations. First, the sample size was small; preferably, we would add additional cases. Second, we did not evaluate CE-MRA, which is known to be useful for follow-ups after stentassisted coil embolization ${ }^{7,18,24}$ and depiction of helmet-type remnants. ${ }^{26}$ Therefore, comparative evaluations with CE-MRA are also required. Third, in this study, we evaluated only anterior circulation aneurysm cases. Additional study is needed because posterior circulation aneurysm cases may yield different results. Fourth, a Pointwise Encoding Time Reduction with Radial Acquisition (PETRA) MRA sequence (Siemens) with similarities to Silent MRA has been reported, ${ }^{28,29}$ and this sequence may yield results similar to those of the present study. A comparison of these sequences should be performed in the future.

We did not set the time interval between the MRA readings. This study may be biased by a neuroradiologist's recognition of previous MRA images. Experienced neuroradiologists might recognize 2 types of MRA images of the same subject. Therefore, this evaluation may, in effect, be nonblinded. Moreover, in this study, there are some of cases of differences in the duration of the period between the evaluation of DSA and MRA images (average, 1.6 days; range, 1-9 days; median, 1 day). These differences may lead to changes in remnant size. 


\section{CONCLUSIONS}

Silent MRA is superior to 3D TOF-MRA in the quality of visualization of a reconstructed artery and has strong concordance with DSA for the detection of aneurysm occlusions. Moreover, Silent MRA may detect helmet-type remnants, thus eliminating the need for gadolinium contrast agents. Silent MRA might therefore be useful for intracranial anterior circulation aneurysms treated with LVIS Jr. stent-assisted coil embolization.

\section{ACKNOWLEDGMENTS}

The authors thank Shuji Sato, Hideo Kawasaki, Issei Fukunaga, Tomoya Muroi, and Haruyoshi Hoshito (Radiologic Technologists, Department of Radiology) for their technical advice.

Disclosures: Nao Takano-RELATED: Grant: Impulsing Paradigm Change through disruptive Technologies Program of the Council for Science, Technology, and Innovation (Cabinet Office, Government of Japan) and Grant-in-Aid for Scientific Research on Innovative Areas, resource and technical support platforms for promoting research "Advanced Bioimaging Support," Comments: This work was funded by the Impulsing Paradigm Change through disruptive Technologies Program of the Council for Science, Technology, and Innovation (Cabinet Office, Government of Japan). This work was also supported by the Japan Society for the Promotion of Science Grantsin-Aid for Scientific Research Grant No. JP16H06280, Grant-in-Aid for Scientific Research on Innovative Areas, resource and technical support platforms for promoting research "Advanced Bioimaging Support"*; UNRELATED: Payment for Lectures Including Service on Speakers Bureaus: GE Healthcare Japan, Comments: 42nd Japanese Society of Radiological Technology Autumn Scientific Congress, October 9-11, 2014, Sapporo, Hokkaido, Japan, and luncheon seminar, "Experience of MR angiography (Silent MRA) using Silenz sequence." Shigeki Aoki-UNRELATED: Board Membership: Bayer Yakuhin; Consulting Fee or Honorarium: GE Healthcare, Comments: luncheon lecture; Grants/Grants Pending: Bayer Yakuhin, Daiich-Sankyo, Eisai, Fuji Seiyaku; Payment for Lectures Including Service on Speakers Bureaus: Bayer Yakuhin, Daiich-Sankyo, Eisai, Fuji Seiyaku, Toshiba, GE Healthcare, Hitachi, Siemens. *Money paid to the institution.

\section{REFERENCES}

1. Molyneux AJ, Kerr RS, Yu LM, et al. International Subarachnoid Aneurysm Trial (ISAT) of neurosurgical clipping versus endovascular coiling in 2143 patients with ruptured intracranial aneurysms: a randomised comparison of effects on survival, dependency, seizures, rebleeding, subgroups, and aneurysm occlusion. Lancet 2005;366:809-17 Medline

2. Pierot L, Spelle L, Vitry F, et al; ATENA Investigators. Immediate clinical outcome of patients harboring unruptured intracranial aneurysms treated by endovascular approach: results of the ATENA study. Stroke 2008;39:2497-504 CrossRef Medline

3. Benitez RP, Silva MT, Klem J, et al. Endovascular occlusion of widenecked aneurysms with a new intracranial microstent (Neuroform) and detachable coils. Neurosurgery 2004;54:1359-67; discussion 1368 CrossRef Medline

4. Fiorella D, Albuquerque FC, Deshmukh VR, et al. Usefulness of the Neuroform stent for the treatment of cerebral aneurysms: results at initial (3-6 mo) follow up. Neurosurgery 2005;56:1191-201; discussion 1201-02 CrossRef Medline

5. Mocco J, Snyder KV, Albuquerque FC, et al. Treatment of intracranial aneurysms with the Enterprise stent: a multicenter registry. J Neurosurg 2009;110:35-39 CrossRef Medline

6. Higashida RT, Halbach VV, Dowed CF, et al. Initial clinical experience with a new self-expanding nitinol stent for the treatment of intracranial cerebral aneurysms: the Cordis Enterprise stent. AJNR Am J Neuroradiol 2005;26:1751-56 Medline

7. Agid R, Schaaf M, Farb R. CE-MRA for follow-up of aneurysms post stent-assisted coiling. Interv Neuroradiol 2012;18:275-83 CrossRef Medline

8. Choi JW, Roh HG, Moon WJ, et al. Optimization of MR parameters of 3D TOF-MRA for various intracranial stents at 3.0T MRI. Neurointervention 2011;6:71 CrossRef Medline
9. Cho WS, Kim SS, Lee SJ, et al. The effectiveness of 3T time-of-flight magnetic resonance angiography for follow-up evaluations after the stent-assisted coil embolization of cerebral aneurysms. Acta Radiol 2014;55:604-13 CrossRef Medline

10. Takayama K, Taoka T, Nakagawa H, et al. Usefulness of contrastenhanced magnetic resonance angiography for follow-up of coil embolization with the Enterprise stent for cerebral aneurysms. J Comput Assist Tomogr 2011;35:568-72 CrossRef Medline

11. Spiotta AM, Miranpuri A, Chaudry MI, et al. Combined balloon stent technique with the Scepter $\mathrm{C}$ balloon and low-profile visualized intraluminal stent for the treatment of intracranial aneurysms. J Neurointerv Surg 2013;5:iii79-82 CrossRef Medline

12. Möhlenbruch M, Herweh C, Behrens L, et al. The LVIS Jr. microstent to assist coil embolization of wide-neck intracranial aneurysms: clinical study to assess safety and efficacy. Neuroradiology. 2014;56: 389-95 CrossRef Medline

13. Behme D, Weber A, Kowoll A, et al. Low-Profile Visualized Intraluminal Support device (LVIS Jr) as a novel tool in the treatment of wide-necked intracranial aneurysms: initial experience in 32 cases. J Neurointerv Surg 2015;7:281-85 CrossRef Medline

14. Ge H, Lv X, Yang X, et al. LVIS stent versus Enterprise Stent for the treatment of unruptured intracranial aneurysms. World Neurosurg 2016;91:365-70 CrossRef Medline

15. Irie R, Suzuki M, Yamamoto M, et al. Assessing blood flow in an intracranial stent: a feasibility study of MR angiography using a Silent Scan after stent-assisted coil embolization for anterior circulation aneurysms. AJNR Am J Neuroradiol 2015;36:967-70 CrossRef Medline

16. Alibek S, Vogel M, Sun W, et al. Acoustic noise reduction in MRI using Silent Scan: an initial experience. Diagn Interv Radiol 2014;20: 360-63 CrossRef Medline

17. Landis JR, Koch GG. The measurement of observer agreement for categorical data. Biometrics 1977;33:159-74 CrossRef Medline

18. Choi JW, Roh HG, Moon WJ, et al. Time-resolved 3D contrastenhanced MRA on 3.0T: a non-invasive follow-up technique after stent-assisted coil embolization of the intracranial aneurysm. $\mathrm{Ko}$ rean J Radiol 2011;12:662-70 CrossRef Medline

19. Takano N, Suzuki M, Irie R, et al. Usefulness of non-contrast-enhanced MR angiography using a Silent Scan for follow-up after Yconfiguration stent-assisted coil embolization for basilar tip aneurysms. AJNR Am J Neuroradiol 2017;38:577-81 CrossRef Medline

20. Penfield JG, Reilly RF. Nephrogenic systemic fibrosis risk: is there a difference between gadolinium-based contrast agents? Semin Dial 2008;21:129-34 CrossRef Medline

21. Kanda $\mathrm{T}$, Ishii $\mathrm{K}$, Kawaguchi $\mathrm{H}$, et al. High signal intensity in the dentate nucleus and globus pallidus on unenhanced T1-weighted MR images: relationship with increasing cumulative dose of a gadolinium-based contrast material. Radiology 2014;270:834-41 CrossRef Medline

22. Yamada N, Hayashi K, Murao K, et al. Time-of-flight MR angiography targeted to coiled intracranial aneurysms is more sensitive to residual flow than is digital subtraction angiography. AJNR Am J Neuroradiol 2004;25:1154-57 Medline

23. Ikushima $Y$, Hashido T, Watanabe $Y$, et al. Effects of imaging parameters on the quality of contrast-enhanced MR angiography of cerebral aneurysms treated using stent-assisted coiling: a phantom study. Magn Reson Med Sci 2017;16:146-51 CrossRef Medline

24. Wang Y, Truong TN, Yen C, et al. Quantitative evaluation of susceptibility and shielding effects of nitinol, platinum, cobalt-alloy, and stainless steel stents. Magn Reson Med 2003;49:972-76 CrossRef Medline

25. van Holten J, Wielopolski P, Brück E, et al. High flip angle imaging of metallic stents: implications for MR angiography and intraluminal signal interpretation. Magn Reson Med 2003;50:879-83 CrossRef Medline

26. Agid R, Willinsky RA, Lee SK, et al. Characterization of aneurysm remnants after endovascular treatment: contrast-enhanced MR an- 
giography versus catheter digital subtraction angiography. AJNR Am J Neuroradiol 2008;29:1570-74 CrossRef Medline

27. Boddu SR, Tong FC, Dehkharghani S, et al. Contrast-enhanced timeresolved MRA for follow-up of intracranial aneurysms treated with the Pipeline embolization device. AJNR Am J Neuroradiol 2014;35: 2112-18 CrossRef Medline

28. Koktzoglou I, Giri S, Piccini D, et al. Arterial spin-labeled carotid
MR angiography: a phantom study examining the impact of technical and hemodynamic factors. Magn Reson Med 2016;75:295-301 CrossRef Medline

29. Grodzki DM, Jakob PM, Heismann B. Ultrashort echo time imaging using Pointwise Encoding Time reduction with Radial Acquisition (PETRA). Magn Reson Med 2012;67:510-18 CrossRef Medline 